\title{
EXISTENCE OF NON-PREPERIODIC ALGEBRAIC POINTS FOR A RATIONAL SELF-MAP OF INFINITE ORDER
}

\author{
EKATERINA AMERIK
}

Let $X$ be a smooth projective variety defined over a number field $K$ and let $f$ : $X \rightarrow X$ be a dominant rational self-map defined over the same number field. As shown in $[\mathrm{AC}]$, one can attach to $f$ a dominant rational map $g: X \rightarrow T$, commuting with $f$ and such that the fiber of $g$ through a sufficiently general complex point $x \in X(\mathbb{C})$ is the Zariski closure of its iterated orbit (or " $f$-orbit") $\left\{f^{k}(x), k \in\right.$ $\left.\mathbb{Z}_{\geq 0}\right\}$. Here "sufficiently general" means "outside of a countable union of proper subvarieties", and so this theorem does not give any information on the $f$-orbits of algebraic points, which, apriori, can have smaller Zariski closure than general complex points. Indeed, the field of algebraic numbers being countable, this countable union of proper subvarieties might contain all the algebraic points of $X$.

One would of course like to show that in reality this never happens and one can always find an algebraic point such that its $f$-orbit is "as large" as the general one. For instance, a conjecture already implicit in $[\mathrm{AC}]$ and formulated by Medvedev and Scanlon in [MS] (Conjecture 5.3) states that if no power of $f$ preserves a non-trivial fibration, then there should be a point $x \in X(\overline{\mathbb{Q}})$ with Zariski-dense $f$-orbit ; a variant of this is an earlier conjecture by S.-W. Zhang stating the same in the case when $f$ is regular and polarized (that is, there is an ample line bundle $L$ on $X$ with $f^{*} L=L^{\otimes q}$ for some $q>1$ ).

What is certainly true in the case when $f$ is regular and polarized is that, at least, there exist points in $X(\overline{\mathbb{Q}})$ with infinite $f$-orbits (that is, non-preperiodic algebraic points). The reason is that in this case, one can introduce the so-called canonical height $\hat{h}_{L}: X(\overline{\mathbb{Q}}) \rightarrow \mathbb{R}$ which is a Weil height function for $L$ with the property $\hat{h}_{L}(f(x))=q \hat{h}_{L}(x)$; it follows that the set of preperiodic points is a set of bounded height and therefore it cannot exhaust $X(\overline{\mathbb{Q}})$ (see $[\mathrm{CS}]$ ). However, the examples of varieties admitting a regular polarized endomorphism are very scarce, so one would like to work in a more general setting; and the theory of canonical heights does not really work for a rational self-map $f$, even if there is an ample line bundle $L$ such $f^{*} L=L^{\otimes q}$ with $q>1$.

The purpose of this note is to provide a short proof of the existence of nonpreperiodic algebraic points for arbitrary dominant rational self-maps of infinite order (Corollary 9), using, though, a result by E. Hrushovski which does not seem to have been treated in a very accessible way at the moment. The argument is very similar to the one used by Bell, Ghioca and Tucker to prove a version of the "dynamical Mordell-Lang conjecture" for étale endomorphisms of quasiprojective varieties; in fact this note is directly inspired by [BGT], and is, in some sense, a continuation of [ABR] where some general observations about iteration of algebraic points by a dominant

Received by the editors November 13, 2010. 
rational self-map with a fixed point have been made. The point is that Hrushovski's result provides periodic points (with good properties) of the reduction of $f$ modulo a suitable prime $\mathfrak{p}$ in the ring of integers of a suitable finite extension of $K$. This means that for some positive integer $k$ one can find an $f^{k}$-invariant $\mathfrak{p}$-adic neighbourhood in $X$ : it is formed by the $\mathfrak{p}$-adic points which reduce modulo $\mathfrak{p}$ to a given point of period $k$. Then one uses an obvious modification of Theorem 3.3 of [BGT] to conclude that all preperiodic points in this neighbourhood are periodic with bounded period $\leq N$ and thus are contained in a certain proper analytic subvariety.

Let us mention that if $f$ were regular, the existence of periodic points modulo $\mathfrak{p}$, i.e. over a finite field, would be very easy; indeed the number of points of $X$ over a finite field is finite, so as soon as $X$ has some points over a finite field $\mathbb{F}_{q}$, all of them are preperiodic for a regular endomorphism defined over $\mathbb{F}_{q}$. But in the case where $f$ is only rational, the existence of such a point is non-trivial because one has to guarantee that not all $\mathbb{F}_{q}$-points eventually land in the indeterminacy locus, as one iterates them by $f$. This is where we use Hrushovski's work (we do not claim, though, that we can avoid it in the regular case, since in the proof of our main result we shall need to avoid the ramification locus as well as the indeterminacy: see Remark 2 at the end of the note). The result we need is as follows:

Theorem 1. ([H], Corollary 1.2) Let $U$ be an affine variety over a finite field $\mathbb{F}_{q}$ and let $S \subset U^{2}$ be an irreducible subvariety over $\overline{\mathbb{F}}_{q}$. Assume that the two projections of $S$ to $U$ are dominant. Denote by $\phi_{q}$ the Frobenius map. Then for any proper subvariety $W$ of $U$, for large enough $m$, there exists $x \in U\left(\overline{\mathbb{F}}_{q}\right)$ with $\left(x, \phi_{q}^{m}(x)\right) \in S$ and $x \notin W$.

(One could of course avoid mentioning $W$ at all, since to produce an $x$ with the required properties and outside of $W$, it suffices to replace $U$ by an affine open subset of $U-W$. But this is the way the result is formulated in $[\mathrm{H}]$ and we, too, want to stress the fact that $x$ can be chosen outside of a proper subvariety since this is exactly what we are going to do.)

In particular, let $\bar{X}$ be any irreducible variety defined over a finite field $\mathbb{F}_{q}$, and let $f: \bar{X} \rightarrow \bar{X}$ be a separable dominant rational self-map defined over the same field. Let $Y=I \cup R$, where $I$ is the indeterminacy locus and $R$ is the ramification locus of $f$. Those are subvarieties defined over a finite extension of $\mathbb{F}_{q}$, and therefore they are periodic under $\phi_{q}$ : for some $k \in \mathbb{N}$ and any $l \in \mathbb{N}, \phi_{q}^{l}\left(I_{j}\right)=\phi_{q}^{l+k}\left(I_{j}\right)$ and $\phi_{q}^{l}\left(R_{j}\right)=\phi_{q}^{l+k}\left(R_{j}\right)$ for all irreducible components $I_{j}$ of $I$ and $R_{j}$ of $R$. Set $V=\bar{X}-Y \cup \phi_{q}(Y) \cup \cdots \cup \phi_{q}^{k-1}(Y)$. Let $U$ be an affine open subset of $V$ defined over $\mathbb{F}_{q}$ and let $S$ be the intersection of $U \times U$ with the graph of $f$. Then by Hrushovski's theorem we have the following

Corollary 2. In the setting as above, there is a point $x \in \bar{X}\left(\overline{\mathbb{F}}_{q}\right)$ such that no iterate $f^{i}(x)$ is an indeterminacy or ramification point of $f$, and $x$ is $f$-periodic. Moreover such points are Zariski-dense in $\bar{X}$.

Let now $X$ be a variety defined over a number field $K^{\prime}$, and let $f: X \rightarrow X$ be a dominant rational self-map defined over $K^{\prime}$. We are going to use corollary 2 to find, for a suitable finite extension $K$ of $K^{\prime}$ and for a suitable prime $\mathfrak{p} \subset \mathcal{O}_{K}$, a "p-adic neighbourhood" in $X$, invariant under some power of $f$, with good properties as in 
$[\mathrm{ABR}]$. The procedure is almost the same as in $[\mathrm{ABR}]$. Take a smooth affine $U \subset X$ such that $f$ is regular on $U$, together with a surjective $K^{\prime}$-morphism $\pi=\left(x_{1}, \ldots, x_{n}\right)$ : $U \rightarrow \mathbb{A}^{n}$ (Noether normalization). Write

$$
\mathcal{O}_{U}=\left(K^{\prime}\left[x_{1}, \ldots, x_{n}\right]\right)\left[x_{n+1}, \ldots, x_{m}\right] / I=K^{\prime}\left[x_{1}, \ldots, x_{n}, x_{n+1}, \ldots, x_{m}\right] / I,
$$

where $I$ contains for instance the minimal polynomials $P_{i}$ of $x_{n+i}, i>0$ over $K\left[x_{1}, \ldots, x_{n}\right]$ (but probably also something else), so that

$$
U=\operatorname{Spec}\left(K^{\prime}\left[x_{1}, \ldots, x_{n}, x_{n+1}, \ldots, x_{m}\right] / I\right) ;
$$

we may suppose that $I$ is given by a system of generators with coefficients from $\mathcal{O}_{K^{\prime}}$ and take a model over $\mathcal{O}_{K^{\prime}}: \mathcal{U}=\operatorname{Spec}\left(\mathcal{O}_{K^{\prime}}\left[x_{1}, \ldots, x_{n}, x_{n+1}, \ldots, x_{m}\right] / I\right)$. By abuse of notation, we denote the rational map on the model by the same $f$. Consider $x_{n+1}, \ldots x_{m}, f^{*} x_{1}, \ldots f^{*} x_{m}$ as power series in $x_{1}, \ldots, x_{n}$. By Lemma 2.1 of $[\mathrm{ABR}]$, their coefficients are $\mathfrak{p}^{\prime}$-integral for almost all primes $\mathfrak{p}^{\prime} \subset \mathcal{O}_{K^{\prime}}$. Choose $\mathfrak{p}^{\prime}$ with this property; and, moreover, such that the minimal monic polynomials $P_{i}$ have $\mathfrak{p}^{\prime}$ integral coefficients and the derivatives $P_{i}^{\prime}$ are not identically zero modulo $\mathfrak{p}^{\prime}$, such that $f$ reduced modulo $\mathfrak{p}^{\prime}$ is well-defined and separable and, finally, such that the reduction $\bar{U}$ modulo $\mathfrak{p}^{\prime}$ :

$$
\bar{U}=\operatorname{Spec}\left(\left(\mathcal{O}_{K^{\prime}} / \mathfrak{p}^{\prime}\right)\left[x_{1}, \ldots, x_{n}, x_{n+1}, \ldots, x_{m}\right] / \bar{I}\right)
$$

is smooth.

The reduction $\bar{U}$ modulo $\mathfrak{p}^{\prime}$ is equipped with a rational self-map $\bar{f}$, the reduction of $f$. By corollary 2, we can find an $\bar{f}$-periodic point $x \in \bar{U}$ over some finite extension $\mathbb{F}_{q}(\alpha)$ (where $\mathbb{F}_{q}=\mathcal{O}_{K^{\prime}} / \mathfrak{p}^{\prime}$ ), such that no iterate of $x$ by $\bar{f}$ is in the indeterminacy or ramification, and such that the values of the derivatives $P_{i}^{\prime}$ modulo $\mathfrak{p}^{\prime}$ calculated at $x$ are non-zero (recall that by construction, the vanishing locus of $P_{i}^{\prime}$ modulo $\mathfrak{p}^{\prime}$ is a proper algebraic subset; the condition on the values of $P_{i}^{\prime}$ at $x$ insures that the injectivity in Proposition 3, (1) below holds true, cf. [ABR]). Let $k$ be the period of $x$, so that $\bar{f}^{k}(x)=x$.

Let $\beta$ be an algebraic number integral over $\mathcal{O}_{K^{\prime}}$ such that the reduction of its minimal monic polynomial over $\mathcal{O}_{K^{\prime}}$ modulo $\mathfrak{p}^{\prime}$ gives the minimal polynomial of $\alpha$, and let $K=K^{\prime}(\beta)$. Let $\mathfrak{p}$ be some prime of $K$ lying over $\mathfrak{p}^{\prime}$. The point $x$ lifts to a point $y \in U\left(K_{\mathfrak{p}}\right)$ by Hensel's lemma (replacing our $(K, \mathfrak{p})$, if necessary, by a slightly larger finite extension of $\left(K^{\prime}, \mathfrak{p}^{\prime}\right)$, we can produce a point $y \in U(K)$ which reduces to $x$, but we won't even need to). Define the $\mathfrak{p}$-adic neighbourhood $O_{\mathfrak{p}, y}$ of $y$ as follows:

$$
O_{\mathfrak{p}, y}=\left\{t \in U\left(K_{\mathfrak{p}}\right) \mid x_{i}(t) \equiv x_{i}(y) \quad(\bmod \mathfrak{p}) \text { for } 1 \leq i \leq m\right\} .
$$

We may suppose that $\pi(y)=(0, \ldots 0) \in \mathbb{A}^{n}$. Then, exactly as in $[\mathrm{ABR}]$, we get the following

Proposition 3. (1) The functions $x_{1}, \ldots x_{n}$ give a bijection between $O_{\mathfrak{p}, y}$ and the $n$-th cartesian power of $\mathfrak{p}$.

(2) The set $O_{\mathfrak{p}, y}$ contains no indeterminacy and no ramification points of $f^{k}$.

(3) $f^{k}\left(O_{\mathfrak{p}, y}\right) \subset O_{\mathfrak{p}, y}$, moreover, $f^{k}$ is bijective on $O_{\mathfrak{p}, y}$.

(4) The $\overline{\mathbb{Q}}$-points of $X$ are dense in $O_{\mathfrak{p}, y}$.

It will be more convenient for us to identify $O_{\mathfrak{p}, y}$ with a cartesian power of $\mathcal{O}_{\mathfrak{p}}$ rather than that of $\mathfrak{p}$. So, if our map $f^{k}$ is given (on the completion of the local ring at $y$ ) by the power series $H_{i}\left(x_{1}, \ldots x_{n}\right)=\left(f^{k}\right)^{*} x_{i} \in \mathcal{O}_{\mathfrak{p}}\left[\left[x_{1}, \ldots x_{n}\right]\right]$, set $F_{i}\left(t_{1}, \ldots, t_{n}\right)=$ 
$\frac{1}{r} H_{i}\left(r t_{1}, \ldots r t_{n}\right)$ where $r$ is some fixed uniformizing element in $\mathcal{O}_{\mathfrak{p}}$. In this way, we may view $O_{\mathfrak{p}, y}$ as $\mathcal{O}_{\mathfrak{p}}^{n}$ with coordinates $t_{i}$, and the map $f^{k}$ is given by the power series $F_{i}$ on $O_{\mathfrak{p}, y}$. Note that, as in [BGT], the $F_{i}$ have integral coefficients (by construction the constant terms of $H_{i}$ are divisible by $r$ ), and moreover the coefficient of $t_{1}^{k_{1}} \ldots t_{n}^{k_{n}}$ in $F_{i}$ is divisible by $r^{k_{1}+\cdots+k_{n}-1}$ when $k_{1}+\cdots+k_{n} \geq 1$ (remark 2.3 of [BGT]).

Write $F=\left(F_{1}, \ldots F_{n}\right): \mathcal{O}_{\mathfrak{p}}^{n} \rightarrow \mathcal{O}_{\mathfrak{p}}^{n}$ (so $F$ is the restriction of $f^{k}$ to the $\mathfrak{p}$-adic neighbourhood, written in coordinates $t_{i}$ ) and consider $F$ modulo $\mathfrak{p}$. Again as in [BGT], we have the following

Proposition 4. There is a positive integer l such that for every $z \in \mathcal{O}_{\mathfrak{p}}^{n}, F^{l}(z) \equiv z$ $(\bmod \mathfrak{p})$.

Proof: Modulo $\mathfrak{p}, F$ is an affine transformation of the linear space $\left(\mathcal{O}_{\mathfrak{p}} / \mathfrak{p}\right)^{n}$. Its linear part $L$ is invertible by Proposition 2.4 of [BGT], since no $f$-iterate of the smooth point $x$ into which our $\mathfrak{p}$-adic neighbourhood reduces modulo $\mathfrak{p}$ is ramification or indeterminacy, and so $\bar{f}^{k}$ is unramified at $x$. Therefore $F$ modulo $\mathfrak{p}$ is an automorphism of a finite-dimensional affine space over a finite field, and some power of it is the identity.

In Section 3 of [BGT], the similar situation is considered; the only difference is that there, the coefficients of all power series are in $\mathbb{Z}_{p}$ rather than in an extension $\mathcal{O}_{\mathfrak{p}}$. The authors prove:

Theorem 5. ([BGT], Theorem 3.3) Let $\phi_{1}, \ldots, \phi_{n} \in \mathbb{Z}_{p}\left[\left[x_{1}, \ldots, x_{n}\right]\right]$ be convergent power series on $\mathbb{Z}_{p}^{n}$ such that $\phi_{i}(x) \equiv x_{i}(\bmod p)$ and the coefficient of $x_{1}^{k_{1}} \ldots x_{n}^{k_{n}}$ in the series $\phi_{i}$ is divisible by $p^{k_{1}+\cdots+k_{n}-1}$ for $k_{1}+\cdots+k_{n}>1$. Let $\omega=\left(\omega_{1}, \ldots \omega_{n}\right) \in \mathbb{Z}_{p}^{n}$. If $p>3$, there exist $p$-adic analytic functions $g_{1}, \ldots g_{n} \in \mathbb{Q}_{p}[[z]]$, convergent on $\mathbb{Z}_{p}$, such that $g_{i}\left(\mathbb{Z}_{p}\right) \subset \mathbb{Z}_{p}, g_{i}(0)=\omega_{i}$ and $g_{i}(z+1)=\phi_{i}\left(g_{1}(z), \ldots, g_{n}(z)\right)$.

They construct $g_{i}(z)$ "by approximation", as a Mahler series

$$
g_{i}(z)=\omega_{i}+\sum_{k=1}^{\infty} b_{i k}\left(\begin{array}{l}
z \\
k
\end{array}\right),
$$

and it turns out, by their construction, that $b_{i k}$ are of the form $\sum_{j=(k+1) / 2}^{\infty} p^{j} c_{i j k}$, where $c_{i j k} \in \mathbb{Z}_{p}$. Since $\left|b_{i k}\right|_{p} \rightarrow 0$ when $k \rightarrow \infty$, these Mahler series define continuous functions on $\mathbb{Z}_{p}$ with values in $\mathbb{Z}_{p}$. To show that these functions are in fact analytic on $\mathbb{Z}_{p}$, one needs to check ([R], Theorem 4.7 of ChapterVI) that $\left|b_{i k}\right|_{p} /|k !|_{p} \rightarrow 0$ when $k \rightarrow \infty$, and this is true for $p>3$ since $\left|b_{i k}\right|_{p} \leq p^{-(k+1) / 2}$ and $1 /|k !|_{p}<p^{k /(p-1)}$.

In the situation when $\Phi=\left(\phi_{1}, \ldots, \phi_{n}\right)$ is an analytic map from $\mathcal{O}_{\mathfrak{p}}^{n}$ to itself and so $\mathbb{Z}_{p}$, not as the domain of definition of $g_{i}$ but as the domain where the $g_{i}$ take their values, is replaced by the extension $\mathcal{O}_{\mathfrak{p}}$, their argument goes through almost verbatim, provided that one replaces $p$ with a uniformizing element $r$ of $\mathcal{O}_{\mathfrak{p}}$ where appropriate: indeed the theory of Mahler series applies to $\mathcal{O}_{\mathfrak{p}}$-valued functions on $\mathbb{Z}_{p}$ as well as to the $\mathbb{Z}_{p}$-valued functions ([R], chapters IV.2.3,VI.4.7). The only adjustment one has to make is in the last step concerning the analyticity of the $\mathcal{O}_{\mathfrak{p}}$-valued map $g_{i}$ on $\mathbb{Z}_{p}$ : instead of being analytic on the whole of $\mathbb{Z}_{p}$, it is analytic on a certain neighbourhood $p^{l} \mathbb{Z}_{p}$. This is because in the expression $b_{i k}=\sum_{j=(k+1) / 2}^{\infty} p^{j} c_{i j k}$, we have to replace $p$ by the uniformizing element $r$, which can be of smaller $p$-adic order $1 / e$; so that $\left|b_{i k}\right|_{p} \leq p^{-(k+1) / 2 e}$ and we need the condition $p>2(e+1)$ in order to guarantee 
$\left|b_{i k}\right|_{p} /|k !|_{p} \rightarrow 0$. But one always has convergence on some $p^{l} \mathbb{Z}_{p}$, where $l$ depends only on $e$ and not on the point $\omega$. Indeed, one has the following elementary lemma:

Lemma 6. A Mahler series $\sum_{k=1}^{\infty} b_{k}\left(\begin{array}{l}z \\ k\end{array}\right)$ defines an analytic function on $p^{l} \mathbb{Z}_{p}$ as soon as

$$
\left|p^{k \frac{p^{l}-1}{(p-1) p^{l}}} b_{k} / k !\right|_{p} \rightarrow 0 .
$$

Proof: Write each term $\left(\begin{array}{c}p^{l} z \\ k\end{array}\right)$ as a product of a constant $C_{k}$ and a polynomial with integral coefficients. As soon as $\left|b_{k} C_{k}\right|_{p} \rightarrow 0$, our Mahler series will be a convergent power series on $p^{l} \mathbb{Z}_{p}$ and thus analytic on $p^{l} \mathbb{Z}_{p}$. A straightforward computation shows that one can take

$$
C_{k}=\frac{1}{k !} p^{l} p^{\left[\frac{k-1}{p}\right]+\left[\frac{1}{p}\left[\frac{k-1}{p}\right]\right]+\cdots+\left[\frac{1}{p}\left[\cdots\left[\frac{k-1}{p}\right] \cdots\right]\right]},
$$

where $[x]$ denotes the integral part of $x$ and in the last summand, the division by $p$ is made $l$ times. The condition $\left|b_{k} C_{k}\right|_{p} \rightarrow 0$ is equivalent to the one in the statement of our lemma: indeed, since we are only interested in the terms which grow together with $k$, we can forget about the integral parts and use the formula for the sum of a geometric progression.

Clearly, the condition in the above lemma is satisfied by the Mahler series $\sum_{k=1}^{\infty} b_{i k}\left(\begin{array}{c}z \\ k\end{array}\right)$ as soon as $l$ is not too small with respect to $e$.

So the theorem of $[\mathrm{BGT}]$ becomes

Theorem 7. Let $\phi_{1}, \ldots, \phi_{n} \in \mathcal{O}_{\mathfrak{p}}\left[\left[x_{1}, \ldots, x_{n}\right]\right]$ be convergent power series such that $\phi_{i}(x) \equiv x_{i}(\bmod \mathfrak{p})$ and the coefficient of $x_{1}^{k_{1}} \ldots x_{n}^{k_{n}}$ in the series $\phi_{i}$ is divisible by $r^{k_{1}+\cdots+k_{n}-1}$ for $k_{1}+\cdots+k_{n}>1$ for a uniformizing element $r$. Let $\omega=\left(\omega_{1}, \ldots \omega_{n}\right) \in$ $\mathcal{O}_{\mathfrak{p}}^{n}$. Then there exist functions $g_{1}, \ldots g_{n}$, continuous on $\mathbb{Z}_{p}$ and analytic on $p^{l} \mathbb{Z}_{p}$ for a certain positive integer $l$ independent of $\omega$, such that $g_{i}\left(\mathbb{Z}_{p}\right) \subset \mathcal{O}_{\mathfrak{p}}, g_{i}(0)=\omega_{i}$ and $g_{i}(z+1)=\phi_{i}\left(g_{1}(z), \ldots, g_{n}(z)\right)$.

Here is an immediate corollary of Theorem 7:

Corollary 8. Let $X, f: X \rightarrow X$ be a variety and a dominant rational self-map defined over a number field. Let $O_{\mathfrak{p}, y}$ be an $f^{k}$-invariant $\mathfrak{p}$-adic neighbourhood constructed in proposition 3 (formed by $K_{\mathfrak{p}}$-points for a suitable extension $K$ of our number field and a suitable prime $\mathfrak{p}$ ). Then there exists a positive integer $N$ such that any preperiodic point in this neighbourhood is periodic of bounded period $\leq N$.

Proof: Indeed, the neighbourhood is $F=f^{k}$-invariant, and some further power $F^{l}$ of $f$ satisfies the conditions for $\phi=\left(\phi_{1}, \ldots, \phi_{n}\right)$ in Theorem 7 . Let $\omega$ be a preperiodic point of $O_{\mathfrak{p}, y}$. From Theorem 7, we deduce that its orbit under a still larger power $\psi=\phi^{s}=f^{N}$, where $N$ depends only on the neighbourhood itself (in particular, on the ramification index of $K_{\mathfrak{p}}$ over $\mathbb{Q}_{p}$ ), is "encoded" by a map $g: \mathbb{Z}_{p} \rightarrow O_{\mathfrak{p}, y}$, analytic on $p^{l} \mathbb{Z}_{p}$, so that this map satisfies $g\left(p^{l} i\right)=\psi^{i}(\omega)$ for $i \in \mathbb{N}$. But an analytic function taking some of its values infinitely many times on $p^{l} \mathbb{Z}$ must be constant, so in fact $\psi(\omega)=\omega$ and so all points with a finite orbit in the neighbourhood are $N$-periodic.

From this, we immediately draw the conclusion announced in the beginning:

Corollary 9. Unless if $f$ is of finite order, there exist points in $X(\overline{\mathbb{Q}})$ which are not preperiodic with respect to $f$. 
Proof: Indeed, points of bounded period must be contained in a proper analytic subvariety of $O_{\mathfrak{p}, y}$, whereas algebraic points are dense in $O_{\mathfrak{p}, y}$.

One can also deduce from the above arguments that such points are even dense in $X$.

Finally, the following remarks are suggested by the referee:

Remark 1 There are several ways to describe a $\mathfrak{p}$-adic neighbourhood, see for example Section 2 of $[\mathrm{BGT}]$ for a "coordinate-free" approach. I have chosen to follow the down-to-earth construction in $[\mathrm{ABR}]$ since it renders the identification of $O_{\mathfrak{p}, y}$ with a cartesian power of $\mathcal{O}_{\mathfrak{p}}$ especially visible.

Remark 2 As mentioned before Theorem 1, we need Hrushovski's result even in the case when $f$ is regular: indeed, in order to satisfy the first condition of Theorem 5 , we need to produce a point $x$ over a finite field such that the powers of the reduction $\bar{f}$ are unramified at $x$. If, for example, $f$ itself is étale, this is possible, and so in this case the proof of the existence of non-preperiodic algebraic points is completely elementary; compare with [BGT] where the "dynamical Mordell-Lang conjecture" is proved for étale endomorphisms.

Acknowledgements: This note has been written during the author's stay at the Institute for Advanced Study. The author has been supported by the NSF grant DMS-0635607 and by the Minerva Research Foundation. I thank the IAS for its hospitality and excellent working conditions, and NSF and Minerva for the support which made my stay possible.

I am grateful to Marat Rovinsky, Antoine Chambert-Loir, Pär Kurlberg, Dragos Ghioca and Tom Tucker for helpful discussions.

\section{References}

[ABR] E. Amerik, F. Bogomolov, M. Rovinsky: Remarks on endomorphisms and rational points, preprint 2010, http://arxiv.org/pdf/1001.1150v2

[AC] E. Amerik, F. Campana: Fibrations méromorphes sur certaines variétés à fibré canonique trivial, Pure Appl. Math. Q. 4 (2008), no. 2 (special issue in honour of F. Bogomolov), part 1, 509-545.

[BGT] J. P. Bell, D. Ghioca and T.J. Tucker: The dynamical Mordell-Lang problem for étale maps, to appear in American J. Math., http://arxiv.org/pdf/0808.3266v1

[CS] G. Call, J. Silverman: Canonical heights on varieties with morphisms, Compositio Math. 89 (1993), 163-205

[H] E. Hrushovski: The elementary theory of the Frobenius automorphism, http://arxiv.org/pdf/math/0406514v1

[MS] A. Medvedev, T. Scanlon: Polynomial dynamics, http://arxiv.org/abs/0901.2352

[R] A. Robert: A course in $p$-adic analysis, Springer Verlag, New York 2000.

[Z] S.-W. Zhang: Distributions in algebraic dynamics, in: Surveys in differential geometry, Vol. X, 381-430, Int. Press, Somerville, MA, 2006.

Université Paris-Sud, Laboratoire de Mathématiques, Campus Scientifique d'Orsay, BÂtiment 425, 91405 Orsay, France.

E-mail address: Ekaterina.Amerik@math.u-psud.fr 